The AstrophysiCAL JoURNAL, 545:1000-1006, 2000 December 20

(C) 2000. The American Astronomical Society. All rights reserved. Printed in U.S.A.

\title{
NO PERSISTENT PULSATIONS IN AQUILA X-1 AS IT FADES INTO QUIESCENCE
}

\author{
A. M. Chandler AND R. E. Rutledge \\ Space Radiation Laboratory, California Institute of Technology, MS 220-47, Pasadena, CA 91125; amc@srl.caltech.edu \\ Received 2000 February 7; accepted 2000 August 7
}

\begin{abstract}
We searched for coherent X-ray pulsations from Aql X-1 in a series of $R X T E$ observations taken shortly after a recent outburst. During the course of these observations, Aql X-1 passes through an apparent "propeller" phase as its luminosity fades to its quiescent value. No pulsations were detected, and we place upper limits (ranging from $0.52 \%$ to $9.0 \%$ ) on the fractional rms amplitude of any periodic signal contained in the various data sets searched. This result has implications for the geometry of the system, if the quiescent luminosity is due to continued low-level accretion. Alternatively, our result supports the idea that the quiescent luminosity may be due to thermal emission.
\end{abstract}

Subject headings: stars: individual (Aquila X-1) - stars: neutron - X-rays: stars

\section{INTRODUCTION}

The origin of the quiescent emission observed from low magnetic field transient neutron stars (NSs) has posed something of a puzzle. When first discovered observationally (in the transient Cen X-4; van Paradijs et al. 1987), the X-ray emission, fitted with a blackbody spectrum, was too faint to have originated over the entire surface of a $10 \mathrm{~km}$ NS. It was suggested that the emission was due to accretion over a fraction (approximately a few percent) of the NS surface. As additional quiescent NS X-ray spectra were observed and fitted with a blackbody spectrum (Verbunt et al. 1994; Asai et al. 1996a, 1996b), the small implied emission areas seemed to confirm this view.

The cause of accretion anisotropy over the surface of a low $B$-field NS has been purported to be the "propeller effect" (Illarionov \& Sunyaev 1975; Stella, White, \& Rosner 1986), in which, at low accretion rates, the magnetic field increases in importance relative to the gravitational field in determining the accretion geometry, perhaps expelling much of the accretion from the system when the effect of the magnetic field is comparable to or stronger than that of the gravitational field at the radius where the Keplerian frequency is equal to the spin frequency of the NS. When this occurs, some fraction of the accretion may follow the magnetic field lines to the magnetic poles which, if these are offset from the rotational poles, could conceivably produce X-ray pulsations. The efficiency of such a propeller has been estimated recently (Menou et al. 1999). However, some observations of decreasing torque with increasing luminosity may indicate deviations from this standard magnetic accretion scenario in low-mass X-ray binaries (Chakrabarty et al. 1997). If, after an outburst, accretion continues into quiescence, then pulsations might be expected, particularly if the NS can be shown to have gone through a "propeller phase," in which the accretion geometry in the vicinity of the NS is dominated by the magnetic field rather than by the gravitational field. No pulsations from these objects in quiescence have yet been reported, although they have been searched for (Verbunt et al. 1994; Asai et al. 1996b).

Thus, until recently, the view of the quiescent luminosity of low- $B$ transient NSs has been as due to continued accretion over a fraction of the surface of the NS, perhaps caused by modified accretion geometry due to the effects of the magnetic field, which may therefore give rise to pulsations in the X-ray intensity.
A different view of the origin of the quiescent luminosity has recently been described (Brown, Bildsten, \& Rutledge 1998), in which nuclear reactions at the base of the NS crust keep the NS core heated to temperatures $\left(\approx 10^{8} \mathrm{~K}\right)$ sufficient to explain a large fraction, if not all, of the quiescent luminosity of these objects $\left(10^{32}-10^{33}\right.$ ergs $\left.{ }^{-1}\right)$; in this manner, the quiescent luminosity can be produced in the absence of active accretion. The discrepancy in the emission area can be explained as due to an incorrect blackbody assumption - specifically, if accretion is shut off, metals stratify in the NS atmosphere (Alcock \& Illarionov 1980; Romani 1987), producing a pure $\mathrm{H}$ photosphere, in which the free-free opacity, which decreases with increasing frequency, dominates, permitting higher $E$ photons to originate at greater depth, thus at higher temperatures (Rajagopal \& Romani 1996; Zavlin, Pavlov, \& Shibanov 1996). This produces an emergent spectrum which is spectrally harder than a simple blackbody and - when described as a simple blackbody - requires a higher $T$ and, subsequently, lower emission area to parameterize it. Spectral fits with $\mathrm{H}$ atmosphere spectra show that the observed X-ray spectra from the three such objects for which data exist (Cen X-4, Aq1 X-1, and 4U 1608 - 522) imply emission areas consistent with a $10 \mathrm{~km}$ NS (Rutledge et al. 1999). Thus, both their quiescent luminosities and their emergent X-ray spectra can be explained in this manner without invoking active accretion during quiescence. An attractive feature of this explanation is that it accounts for the similar luminosities of these three objects without requiring their quiescent mass accretion rates (a product of the propeller efficiency and mass accretion rate through the accretion disk) to be serendipitously similar.

It remains an open question whether or not accretion (at a level of $\dot{M} \lesssim 10^{-12} M_{\odot} \mathrm{yr}^{-1}$ ) occurs at all, contributing some fraction of the quiescent X-ray luminosity. There are few observational means by which this may be investigated. One such possibility is to search for metal lines in the quiescent X-ray spectrum, which, if present, would imply that metals are being fed into the atmosphere at a rate greater than gravity can stratify it (Bildsten, Salpeter, \& Wasserman 1992; see Zavlin et al. 1996 for X-ray spectra of metallic atmospheres). Such spectra can be investigated by the latest generation of X-ray spectroscopy missions $(A X A F$ and $X M M)$. A second method is to search for intensity variability during quiescence. In particular, as described 
above, if pulsational variability at a particular frequency were to be found in quiescence, this would support the scenario of active accretion along the magnetic field lines to the NS surface. Alternatively, if the NS magnetic field were strong enough to significantly affect the photospheric opacity ( $>10^{10} \mathrm{G}$; see Zavlin et al. 1995), this would produce apparent "hot spots" near the magnetic poles of the NS which, if offset from the rotation axis, could give rise to pulsations; however, it is thought that type I X-raybursting sources have magnetic fields below this value. Clearly, discovery of pulsations or constraints on the pulsed fraction during X-ray quiescence (following the onset of a propeller) would provide useful limits for models of quiescent NS emission.

The system Aq1 X-1 lends itself to such a search for pulsations. During the X-ray decline of a recent outburst, the intensity at first decreased by 1 order of magnitude over 17 days, then abruptly (beginning at $L_{\mathrm{X}} \approx 10^{36} \mathrm{ergs} \mathrm{s}^{-1}$ ) by 3 orders of magnitude over 3 days, which was coupled with the sudden spectral hardening of the X-ray intensity (Campana et al. 1998; Zhang, Yu, \& Zhang 1998a). The X-ray intensity stopped dropping at a luminosity $L_{\mathrm{X}} \approx 10^{33}$ ergs s${ }^{-1}$, comparable to the quiescent luminosity observed previously (Verbunt et al. 1994). This was interpreted as evidence for a propeller phase, though this remains to be confirmed through repeated observations of Aql X-1 in outburst. If indeed this behavior does indicate a propeller phase in Aq1 X-1, it offers the opportunity to search for pulsations during the period when the NS magnetic field substantially alters the accretion geometry.

Here we have searched for, and not found, pulsations in Aq1 X-1 during and immediately following its supposed propeller phase. In $\S 2$ we describe our search method and the results of our analysis. In $\S 3$ we discuss these results and our conclusions.

\section{ANALYSES}

\subsection{Search Methodology}

We used high time resolution data taken with $R X T E$ PCA (Swank et al. 1996). The PCA detectors have a total geometric area of $6500 \mathrm{~cm}^{2}$ and a nominal energy range of 2-60 keV. We selected for analysis four observations, based on the results of Zhang et al. (1998b). These were listed by Zhang et al. as observations $8,10,11$, plus a later observation, 12 , which was not analyzed by Zhang et al. due to the faintness of the source-which made their spectral analysis infeasible, but it can still be useful in our search for pulsations. Based on the results of Zhang et al., the beginning of the propeller phase appears to be between observations 9 and 10, and so our timing analyses of observations 10-12 are expected to reveal pulsations created by the magnetic field-modified accretion geometry (if any), while observation 8, from when the source was moderately brighter, is included simply for comparison purposes. We list details of the observations in Table 1. Data were obtained from the $X T E$ archive at HEASARC.

For our data analysis, we used the standard FTOOLS/ XTE v4.0. Using standard 2-formatted data, we extracted the X-ray spectrum from each observation and subtracted background counts (estimated using the pcabackest tool), and examined the counts spectra. Based on the spectrum of the faintest observation (number 12), which becomes background-dominated above $\sim 12 \mathrm{keV}$, we selected for our analysis pulse-height analyzer (PHA) bins 0-20, corresponding in energy roughly to $2-12 \mathrm{keV}$. We then extracted individual photon events from data in E125 mode (which has time resolution of $\sim 125 \mu \mathrm{s}$ ) and corrected the photon arrival times (times of arrival, TOAs) to the solar system barycenter.

Zhang et al. (1998b) reported strong evidence for a 549 $\mathrm{Hz}$ pulsation frequency, observed during $\sim 10 \mathrm{~s}$ following a type I X-ray burst from Aql X-1. (It is possible that this may be the second harmonic of the NS spin frequency.) Similar pulsations have been observed from type I bursts of five other sources $(1728-34$, Strohmayer et al. 1996; 1636-53, Zhang et al. 1996; 1731-260, Smith, Morgan, \& Bradt 1997; a galactic center source, possibly MXB 1743-29, Strohmayer et al. 1997; and 1702-43, Markwardt, Strohmayer, \& Swank 1999). Rather than restrict our efforts to a small range of frequencies around the previously measured value, we chose to do a more general search for pulsations over the broad frequency range $0.5-1024 \mathrm{~Hz}$. This range includes all previously reported rotation frequencies for type I X-ray bursters. We were able to do this more general search without a significant loss of sensitivity for reasons which we explain below, in $\S 2.4$.

The Aql X-1 system consists of an NS and a companion star, both orbiting the system's center of mass with a period of about $19 \mathrm{hr}$ (see $\S 2.2$ below). As a result of this orbital motion, the NS is accelerating along our line of sight. Any pulsations will therefore be observed with a Doppler-shifted frequency which is not constant in time. In a standard fast Fourier transform (FFT) analysis, the spectral power resulting from such a signal may be spread out over many frequency bins, drastically decreasing the probability of detection. For example, consider a $550 \mathrm{~Hz}$ pulsar in a $19 \mathrm{hr}$ circular orbit with a maximum projected velocity of $100 \mathrm{~km}$ $\mathrm{s}^{-1}$. If we calculate a coherent power spectrum using $T=2048 \mathrm{~s}$ of data, the power from this pulsar will be spread over as many as 70 independent frequency bins (for the worst case orbital phase, the observed pulsar frequency covers a range $\Delta f=0.034 \mathrm{~Hz}$, giving $T \Delta f=70$ bins). To counteract this effect, we attempt to remove the acceleration of the pulsar signal in the time domain before performing the FFT. Because we do not have complete knowledge of

TABLE 1

$R X T E$ Aquila X-1 Analyzed Observations

\begin{tabular}{cccr}
\hline \hline Observation & $\begin{array}{c}\text { Start Time/End Time } \\
(\text { UT })\end{array}$ & $\begin{array}{c}\text { Start Time } \\
(\text { JD })\end{array}$ & $\begin{array}{c}\text { Start Time } \\
(\text { MET })\end{array}$ \\
\hline 20098-03-08-00..... & 1997 Mar 01 21:33/1997 Mar 02 00:21 & $2,450,509.398$ & $99,869,572$ \\
$20098-03-10-00 \ldots \ldots$ & 1997 Mar 05 22:55/1997 Mar 06 01:28 & $2,450,513.456$ & $100,220,227$ \\
$20098-03-11-00 \ldots \ldots$. & 1997 Mar 08 21:31/1997 Mar 09 01:11 & $2,450,516.394$ & $100,474,022$ \\
$20098-03-12-00 \ldots \ldots$ & 1997 Mar 10 21:25/1997 Mar 10 23:55 & $2,450,518.389$ & $100,646,431$ \\
\hline
\end{tabular}


Aql X-1's orbital parameters, we must cover the possible orbital parameter space with a number of acceleration trials and repeat the Fourier analysis for each.

For our purposes, the orbit (assumed circular) can be characterized by three independent parameters - the orbital period $P_{\text {orb }}$, the projected circular velocity $v$, and the orbital phase $x_{0}$ of the NS at the start of the observation in question. The projected circular velocity is the magnitude of the NS's circular velocity projected along our line of sight, $v=$ $v_{\text {NS }} \sin i$, where $i$ is the angle between the line of sight and the normal to the plane of the orbit. Note that we could equally well have chosen the projected orbital radius $\left(a \sin i=v P_{\text {orb }} / 2 \pi\right)$ in lieu of this parameter. The orbital phase $x_{0}$ is measured in cycles and is therefore in the range $[0,1]$, with $x_{0}=0$ corresponding to longitude $=0$ (where the observed pulsar frequency would be measured at its minimum). The signal from a pulsar in such an orbit would be observed with a Doppler-shifted frequency

$$
f(t)=f_{0}\left[1-\frac{v}{c} \cos \left(\frac{2 \pi}{P_{\text {orb }}} t+2 \pi x_{0}\right)\right],
$$

where $f_{0}$ is the rest frequency of the pulsar, $c$ is the speed of light, and $t$ is the elapsed time since phase $x_{0}$.

Pulsar orbital acceleration searches are typically carried out (see, for example, Anderson et al. 1990) by correcting data with an assumed constant acceleration $f(t)=f_{0}+\dot{f t}$. This is applicable when the data cover only a small part of the pulsar's orbit, or the pulsar is very strong. In the first case, the short span of orbit is well approximated by a constant acceleration, while in the second case, some spreading of the feature in the power spectrum can be tolerated without the signal disappearing into the noise. For the Aql X-1 searches reported here, we assumed that neither of these conditions was satisfied. Our acceleration searches therefore fully correct for an assumed circular orbit $\left(P_{\text {orb }}, v, x_{0}\right)$. As compared with the constant acceleration method, this circular acceleration method may require more trial accelerations to cover a given orbital phase space, but the detection significance is greatly increased. This is because the circular method recovers significantly more signal power in a single power spectrum bin, more than making up for the increased number of trials (the detection significance is exponential in recovered power but only linear in the number of trials).

After preparing an energy-selected, barycentered-photon TOA list, we proceeded with the search method as follows: First, we assumed particular values for the orbital parameters (i.e., a trial acceleration) from within the search phase space (of orbital period, velocity, and initial phase; see Table 2). We corrected the TOAs for the assumed acceleration by introducing a corrected time $\tilde{t}$, which is a function of the original time $t$, such that the frequency as a function of $\tilde{t}$ is constant. Equivalently, we require the integrated

TABLE 2

Searched Parameter Space

\begin{tabular}{cc}
\hline \hline Parameter & Parameter Space \\
\hline$P_{\text {orb }}(\mathrm{hr}) \ldots \ldots \ldots \ldots \ldots \ldots \ldots \ldots \ldots$ & $18.91-19.45$ \\
$v_{\text {NS }} \sin i\left(\mathrm{~km} \mathrm{~s}^{-1}\right) \ldots \ldots \ldots \ldots \ldots$. & $0-130$ \\
Initial orbital phase $($ cycles $) \ldots \ldots$. & $0-1$ \\
Pulsar spin frequency $(\mathrm{Hz}) \ldots \ldots$. & $0.5-1024$ \\
\hline
\end{tabular}

phase to be linear in $\tilde{t}$ :

$$
x(t)-x_{0}=\int_{0}^{t} f\left(t^{\prime}\right) d t^{\prime}=f_{0} \tilde{t} .
$$

Integrating equation (1) for a given acceleration trial $\left(P_{\text {orb }}, v, x_{0}\right)$, we see that the $i$ th TOA $t_{i}$ is corrected to

$$
\tilde{t}_{i}=t_{i}+\frac{\beta}{\omega}\left[\sin \left(2 \pi x_{0}\right)-\sin \left(\omega t_{i}+2 \pi x_{0}\right)\right],
$$

where $\beta=v / c$ and $\omega=2 \pi / P_{\text {orb }}$.

These corrected TOAs were used to construct a time series which was then FFTed and used to produce an estimate of the power density spectrum (PDS). The PDS was searched for candidates (frequency bins containing statistically significant excess power). The process was repeated for each acceleration trial. The spacing of acceleration trials in (orbital period, velocity, and phase) parameter space was chosen to allow sensitivity to as weak a signal as possible while keeping the computational requirements reasonable.

\subsection{Determination of Searched Parameter Space}

Similar, but inconsistent, values for the orbital period of Aq1 X-1 have been published by two groups. Based on observations of Aql X-1 during outburst, Chevalier \& Ilovaisky (1991) measured the orbital period to be $18.97 \pm 0.02 \mathrm{hr}$. Shahbaz et al. (1998) determined the orbital period in quiescence to be $19.30 \pm 0.05 \mathrm{hr}$. In a more recent analysis, Chevalier \& Ilovaisky (1998) report the period to be $18.9479 \pm 0.0002 \mathrm{hr}$, again measured during outburst, but they also report a quiescent period within $0.02 \%$ of this value. To be safe, we chose to cover a range of orbital periods that encompasses a $3 \sigma$ range in all of these measurements (as well as the periods in between):

$$
18.91 \mathrm{hr}<P_{\text {orb }}<19.45 \mathrm{hr} \text {. }
$$

Fortunately, we were able to cover this entire range with one trial value of $P_{\text {orb }}$ (see $\S 2.3$ below).

The projected circular velocity $v$ has not been measured. We can determine an upper limit on $v$ for our search by assuming a value for the NS mass, $m_{\mathrm{NS}}=1.4 M_{\odot}\left(M_{\odot}=\right.$ one solar mass), and choosing a maximum companion mass to which we will be sensitive. We can then use our knowledge of the orbital period to calculate the orbital velocity of the NS. Recently, the true optical counterpart of Aq1 X-1 has been identified as a late K-type star (Chevalier et al. 1999). Again, to be safe we decided to cover a range of velocities corresponding to a companion star mass as high as $1.0 M_{\odot}$ (for all possible orbital inclination angles). This results in a search range of

$$
0 \mathrm{~km} \mathrm{~s}^{-1} \leq v \leq 130 \mathrm{~km} \mathrm{~s}^{-1} \text {. }
$$

Note that our search was also sensitive to larger companion masses in a restricted range of inclination angles; e.g., our search covered companion masses up to $2.0 M_{\odot}$ for $0^{\circ} \leq i \leq 40^{\circ}$.

For the starting orbital phase for each observation, rather than rely on a particular ephemeris, we simply chose to search the full range

$$
0 \text { cycles } \leq x_{0} \leq 1 \text { cycle . }
$$

Our search phase space is summarized in Table 2. 


\subsection{Searches Performed}

The $R X T E$ observations included in this analysis were roughly $9-13 \mathrm{ks}$ in duration. The TOA data in each observation are broken up into two or three cohesive sections, separated by gaps due to Earth occultations. Each continuous section is at least $2048 \mathrm{~s}$ long, and we chose to study these continuous blocks individually. Observation 10, with one occultation dropout, is therefore divided into two sections, which we call observations $10 \mathrm{a}$ and $10 \mathrm{~b}$. Observations 11 and 12 are similarly divided (into $11 \mathrm{a}, 11 \mathrm{~b}, 11 \mathrm{c}$, $12 \mathrm{a}$, and $12 \mathrm{~b}$ ). We analyzed only the first section of observation 8 , for a total of eight separate data sets. The photons from a given data set were binned into a $2^{23}$ point time series, with a time resolution of $\Delta t=244 \mu \mathrm{s}$. Over the course of the observations, the source count rate decreases from about 670 counts $\mathrm{s}^{-1}$ at the beginning of observation 8 to less than 10 counts $\mathrm{s}^{-1}$ by the end of observation 12 , with a background rate of about $31-37$ counts $\mathrm{s}^{-1}$ (see Table 3).

Our search method was described above in $\S 2.1$. We now describe in detail the method used to determine the grid of trial values for the orbital parameters. Since we must discretize a continuous phase space, there will likely be some offset between a signal's actual parameters and our nearest trial values. The effect of this offset will be some spreading out of the signal power in the power spectrum, since the time dependence of the signal's frequency will not have been completely removed. In choosing the orbital trials, the general idea was to space them just finely enough to keep the remaining frequency drift within tolerable limits (to be quantified below).

We began analytically and then made some empirical adjustments. Considering each of the three orbital parameters $\left(P_{\text {orb }}, v, x_{0}\right)$ separately, we took partial derivatives of equation (1) to determine the frequency drift that results from offsets in the individual parameters. For example, an error in the velocity $(\partial v=c \partial \beta)$ causes a drift of

$$
\partial f=f_{0} \cos \left(\omega t+2 \pi x_{0}\right) \partial \beta .
$$

We are interested only in the extrema of the frequency range covered by the signal over the course of the observation time $T$, and for simplicity, we chose to make our velocity trial values independent of the other parameters. We therefore replace the cosine factor in equation (7) by its maximum change over the course of an observation $(T=2048 \mathrm{~s})$, and we replace $f_{0}$ by our maximum search frequency $\left(f_{0} \rightarrow 1024 \mathrm{~Hz}\right)$. The maximum frequency drift caused by a velocity offset is therefore

$$
\partial f_{\max }=(1024)(0.19) \partial \beta \mathrm{Hz} .
$$

A "tolerable" drift must be no greater than the independent frequency resolution of the power spectrum, $F$. Since we are considering each orbital parameter separately, for these initial calculations we restrict the error-induced drift from each to $F / 2$. This is somewhat arbitrary, and the actual spacings used were decided on with the help of simulations. Note that the allowable spacing of the trials can be twice the maximum allowable offset. We now have for the velocity trial spacing

$$
\Delta v_{\text {trial }} \lesssim \frac{c F}{10240.19}=0.77\left(\frac{F}{5 \times 10^{-4} \mathrm{~Hz}}\right) \mathrm{km} \mathrm{s}^{-1} .
$$

For a coherent FFT of $2048 \mathrm{~s}$ of data, the independent Fourier resolution is $F=1 / T=4.88 \times 10^{-4} \mathrm{~Hz}$. The search strategy that we settled on actually used a larger frequency resolution (see below); for now we will leave the expressions for the trial spacings in terms of $F$ explicitly.

Again, for simplicity, we chose to keep the spacing of the $P_{\text {orb }}$ and $x_{0}$ trials independent of $P_{\text {orb }}$ and $x_{0}$, but we did allow for velocity dependence. For the orbital period, we ultimately find

$$
\Delta P_{\text {trial }} \lesssim 2800\left(\frac{F}{5 \times 10^{-4} \mathrm{~Hz}}\right)\left(\frac{v}{100 \mathrm{~km} \mathrm{~s}^{-1}}\right)^{-1} \mathrm{~s} .
$$

Even for the smallest Fourier resolution $\left(F=4.88 \times 10^{-4}\right.$ $\mathrm{Hz})$ and the largest velocity in our search range $(v=130 \mathrm{~km}$ $\mathrm{s}^{-1}$ ), the orbital period trial spacing turns out to be larger than our target search range of $(19.45-18.91 \mathrm{hr})(3600 \mathrm{~s}$ $\left.\mathrm{hr}^{-1}\right)=1944 \mathrm{~s}$. In other words, a single trial value was sufficient to cover our entire search range in $P_{\text {orb }}$. Thus, we did not actually search over trial values of the orbital period. For the initial orbital phase trial spacing, we find

$$
\begin{aligned}
\Delta x_{\text {trial }} \lesssim & 1.2 \times 10^{-3}\left(\frac{F}{5 \times 10^{-4} \mathrm{~Hz}}\right) \\
& \times\left(\frac{v}{100 \mathrm{~km} \mathrm{~s}^{-1}}\right)^{-1} \text { cycles } .
\end{aligned}
$$

\begin{tabular}{|c|c|c|c|c|}
\hline Observation & MET Analyzed & Source Count Rate ${ }^{a}$ & Background Count Rate ${ }^{\mathrm{a}, \mathrm{b}}$ & $\begin{array}{c}\text { Fractional RMS Amplitude }{ }^{c} \\
(\%)\end{array}$ \\
\hline $8 a \ldots \ldots \ldots . .$. & $99,869,572-98,871,620$ & $670.6 \pm 0.8$ & 37.3 & $<0.52$ \\
\hline $10 \mathrm{a} \ldots \ldots \ldots$ & $100,220,227-100,222,275$ & $188.2 \pm 0.6$ & 35.8 & $<1.0$ \\
\hline $10 \mathrm{~b} \ldots \ldots \ldots . .$. & $100,226,227-100,228,275$ & $177.4 \pm 0.6$ & 35.8 & $<1.1$ \\
\hline $11 \mathrm{a} \ldots \ldots \ldots \ldots$ & $100,474,022-100,476,070$ & $52.6 \pm 0.5$ & 35.7 & $<2.3$ \\
\hline $11 \mathrm{~b} \ldots \ldots \ldots . .$. & $100,479,522-100,481,570$ & $48.6 \pm 0.5$ & 35.7 & $<2.5$ \\
\hline $11 \mathrm{c} \ldots \ldots \ldots \ldots$ & $100,485,082-100,487,130$ & $45.5 \pm 0.5$ & 35.7 & $<2.6$ \\
\hline $12 \mathrm{a} \ldots \ldots \ldots$ & $100,646,431-100,648,479$ & $19.0 \pm 0.5$ & 31.2 & $<4.9$ \\
\hline $12 b \ldots \ldots \ldots$ & $100,652,431-100,654,479$ & $9.2 \pm 0.5$ & 31.2 & $<9.0$ \\
\hline
\end{tabular}

If we were to use these spacings with coherent $2048 \mathrm{~s}$ FFTs, each of the eight data sets would require over 95,000

TABLE 3

ObSERVATION Parameters and Search Results

${ }^{\text {a }}$ Counts $\mathrm{s}^{-1}\left(13,000\right.$ counts $\left.\mathrm{s}^{-1}=1 \mathrm{crab}\right)$.

${ }^{b}$ Systematic uncertainty $\sim 0.5$ counts $\mathrm{s}^{-1}$.

${ }^{c}$ Upper limits are $95 \%$ confidence. 
trial accelerations. This would require $\gtrsim 100$ days of CPU time on a Sun Ultra 10 workstation. To reduce the computational requirements, we chose to utilize incoherently stacked power spectra. The original data of duration $T$ are divided into $\mathscr{S}$ sections of duration $T / \mathscr{S}$. Each section is FFTed individually, and the $\mathscr{S}$ individual power spectra are (incoherently) added together. The independent Fourier step size has been increased by a factor of $\mathscr{S}[F=1 /(T /$ $\mathscr{S})=\mathscr{S} / T]$, and the trial parameter spacings increase by the same factor. The total number of trials is therefore reduced by a factor of $\mathscr{S}^{2}$ (since we are searching over two parameters). The reduction in the number of trials comes at the cost of reduced sensitivity, so the number of stacks $\mathscr{S}$ should be kept as small as possible, just barely bringing the number of trials to a feasible level. For our analyses, we chose to use $\mathscr{S}=4$.

With the above analytical calculations as guides, we used simulations to determine the trial spacings actually used in the search. Our simulations upheld the decision to use a single $P_{\text {orb }}$ trial. Since there are indications that the orbital period is more likely to lie toward the lower end of our search range (18.91-19.45 hr), we chose to use $P_{\text {orb }}=19.00$ $\mathrm{hr}$ in our trials. We decided to use a velocity trial spacing of

$$
\Delta v_{\text {trial }}=2.0 \mathrm{~km} \mathrm{~s}^{-1} \text {. }
$$

This is about 1.5 times finer than the spacing calculated above (recall that we are using $\mathscr{S}=4$ stacks). For the orbital phase, we decided on a trial spacing of

$$
\Delta x_{\text {trial }}=0.011\left(\frac{v}{100 \mathrm{~km} \mathrm{~s}^{-1}}\right)^{-1} \text { cycles, }
$$

which is about 2.3 times coarser than the spacing indicated in the analytical calculation. For small velocities, we used a minimum of $16 x_{0}$ trials (except for the single $v=0$ trial).

Using these spacings with 2 times-oversampled power spectra (exactly like our actual searches), we determined that, at least $98 \%$ of the time, we were able to recover at least $77 \%$ of a simulated signal's power in a single spectral bin, even in the least favorable regions of our search phase space. We simulated signals whose orbital parameters were offset from our search trials and whose pulsation frequencies were offset from our discrete Fourier frequencies. The reduction in recovered signal power is due to a combination of these factors. (Note that it is mere coincidence that a simple FFT recovers, on average, $77 \%$ of a signal's power based solely on offset from the discrete Fourier frequencies. In a 2 times oversampled-spectrum, the minimum power recovered, based solely on frequency offset, is $81 \%$.) Our peak detected power was not always in the frequency bin closest to the rest frequency of the simulated pulsar. This has no effect on the detection of pulsations; the true frequency and orbit of the pulsar can be refined after the initial detection.

In total, we covered the orbital phase space with 4069 acceleration trials. The same accelerations were used for each of the eight data sets.

\subsection{Estimation of Detection Sensitivities}

To characterize the sensitivity of our search, we wished to place quantitative limits on the minimum signal strength required for a significant detection. Since the noise statistics of our power spectra are well understood, it is a simple matter to determine the detection threshold, the minimum spectral power $P_{\text {det }}$ required for a detection. This information can be used to determine the minimum required signal strength. For a review of detection thresholds, detection sensitivities, and upper limits, see Vaughan et al. (1994).

Our stacked power spectra were constructed by summing four individual spectra, each normalized to a mean power of 1 [note that this convention differs from the commonly used Leahy normalization (Leahy et al. 1983), for which the mean noise power is 2]. In the absence of a signal, the power $P$ in a given spectral bin follows a $\chi^{2}$ distribution. Specifically, $2 P$ is $\chi^{2}$ distributed with $2 \mathscr{S}=8$ degrees of freedom. The probability that the power $P$ in a single PDS bin will exceed a given value $P_{0}$ is therefore

$$
p\left(P>P_{0}\right)=e^{-P_{0}}\left(1+P_{0}+\frac{1}{2} P_{0}^{2}+\frac{1}{6} P_{0}^{3}\right) .
$$

Considering each frequency bin of each PDS to be one search "trial," our entire search consisted of $N_{\text {trials }}=3.4$ $\times 10^{10}$ such trials. This is the product of the number of observations searched (8), the number of acceleration trials for each observation (4069), and the number of frequencies searched in each power spectrum $\left(2^{20}\right)$. The latter number is equal to the frequency range searched $(1024 \mathrm{~Hz})$ divided by the independent frequency resolution $[F=(\mathscr{S} / 2048) \mathrm{Hz}]$ times the oversampling factor (2). Of course, neighboring bins in an oversampled spectrum are not independent, and neighboring acceleration trials may not produce truly independent spectra. Thus, $N_{\text {trials }}$ is an upper limit to the number of statistically independent trials in the search. Since we are overestimating the number of independent trials, our detection threshold will be conservative.

The statistical significance $S$ of a measured power $P_{0}$ is equal to the probability that the power was produced by a random noise fluctuation. For small $p$ (large powers), the significance is

$$
S=N_{\text {trials }} p\left(P>P_{0}\right) .
$$

To achieve our target significance of $10^{-4}$, we require a PDS power of at least

$$
P_{\text {det }}=43 \text {. }
$$

For a given data set containing $N_{t}$ total counts, $N_{s}$ of which were emitted by the source, we can relate the source strength to the expected spectral power by

$$
\langle P\rangle=4+\frac{1}{4} \frac{N_{s}^{2}}{N_{t}} \mathscr{F}^{2}
$$

(Buccheri, Sacco, \& Ozel 1987; van der Klis 1989), where $\mathscr{F}$ is the pulsed fraction, i.e., the fraction of source counts that actually contribute to the pulsation. The first term in equation (17) is the expected noise power $\left(\left\langle P_{N}\right\rangle=4\right.$ because we have summed four power spectra, each normalized to unity), while the second term is the expected signal power. Here, we have assumed that the signal waveform is sinusoidal. $N_{s}$ and $N_{t}$ are determined for each data set before beginning the pulsation search. Therefore, the sensitivity of each search is determined as a limit on the pulsed fraction $\mathscr{F}$.

We now calculate the detection sensitivities for the eight data sets searched. For a given observation, the detection sensitivity is expressed as the minimum pulsed fraction required to produce a spectral power exceeding $P_{\text {det }}$ with 
high confidence. Instead of simply solving equation (17) for $\mathscr{F}$ using the detection threshold power $P_{\text {det }}=43$, we must allow for statistical variation of the spectral power produced by a source of a given strength, as well as variation in the recovered power due to the discrete nature of the search trials.

To determine the detection sensitivity, we must consider the probability distribution of power in a spectral bin containing a signal plus noise. If, in the absence of noise, the signal produces a power $P_{\text {sig }}$, then the power $P$ in the presence of noise will be distributed according to

$$
\begin{aligned}
p_{n}\left(P>P_{0} ; P_{\text {sig }}\right)= & \exp \left[-\left(P_{0}+P_{\text {sig }}\right)\right] \\
& \times \sum_{m=0}^{\infty} \sum_{k=0}^{m+n-1} P_{0}^{k} P_{\text {sig }}^{m} /(k ! m !)
\end{aligned}
$$

(Groth 1975), where $n$ is the number of independent bins that were summed to produce $P$ (i.e., in our case $n=\mathscr{S}=4)$. Thus, $p_{n}\left(P>P_{0} ; P_{\text {sig }}\right)$ is the probability that the power will exceed $P_{0}$ in the spectral bin containing the signal. It is important to note that noise power and signal power are not simply additive; $P$ will not always exceed $P_{\text {sig }}$ (see Vaughan et al. 1994 for a discussion of this point). For a given $N_{s}$ and $N_{t}$, we would like to find the pulsed fraction $\mathscr{F}$ that is $95 \%$ likely to produce power $P>P_{\mathrm{det}}$.

To ensure that our reported sensitivity is as conservative as possible, we will not naively invert equation (18) for $P_{\text {sig }}$ with $P_{0}=P_{\text {det. }}$. Instead, we consider the worst case scenario covered by our search parameter space. Due to the discrete binning of photons in the construction of the time series, the recovered signal power in the FFT falls off with increasing frequency. A signal near our maximum search frequency$1024 \mathrm{~Hz}$, which is half the Nyquist frequency of our power spectra-will produce $81 \%$ of the power that would be recovered from a low-frequency signal of the same intrinsic strength. Also, due to our discrete grid of acceleration trials and frequency trials, we are only $98 \%$ likely to recover more than $77 \%$ of a signal's available power. Thus, in our worst case, we are $98 \%$ likely to recover at least $(0.77)(0.81) P_{\text {sig }}=$ $0.62 P_{\text {sig. }}$.

We account for this reduction in recovered signal power approximately by solving equation (18) in the form

$$
p_{n}\left(P>P_{\text {det }} ; 0.62 P_{\text {sig }}\right)=0.97 \text {. }
$$

The probability of our recovered signal power exceeding $0.62 P_{\text {sig }}$ and our total spectral power $P$ exceeding $P_{\text {det }}$ is then approximately $(0.98)(0.97) \approx 0.95$, giving our desired $95 \%$ confidence.

Solving equation (19) (numerically) results in $P_{\text {sig }}=93.6$. For a given observation, the minimum required pulsed fraction is then given by $\mathscr{F}=\left(4 N_{t} P_{\text {sig }} / N_{s}^{2}\right)^{1 / 2}$ and converted to rms by dividing by $2 \sqrt{2}$. Thus, our $95 \%$ confidence detection sensitivities for observations $8 \mathrm{a}, 10 \mathrm{a}, 10 \mathrm{~b}, 11 \mathrm{a}, 11 \mathrm{~b}, 11 \mathrm{c}$, $12 \mathrm{a}$, and $12 \mathrm{~b}$ are given by $0.60 \%, 1.2 \%, 1.2 \%, 2.7 \%, 2.9 \%$, $3.0 \%, 5.6 \%$, and $10 \%$, respectively. We verified these numbers with Monte Carlo simulations.

Our search included spin frequencies up to $1024 \mathrm{~Hz}$. Had we restricted our search to a smaller range of frequencies, corresponding to the previously reported pulsations, our detection sensitivities would not have been drastically different. To achieve the same detection significance, with far fewer trials, we require only a slightly reduced spectral power. And since the pulsed fraction limit depends essentially on the square root of the required power, the detec- tion sensitivities are not terribly sensitive to the number of frequency trials in the search. Given Fox's best estimate for the pulsation frequency $549.76_{-0.03}^{+0.05} \mathrm{~Hz}$ (D. Fox 1999, private communication), if we had confined our search to a $5 \sigma$ range (about $549.76 \mathrm{~Hz}$ and $274.88 \mathrm{~Hz}$ ), our detection sensitivity in observation $10 \mathrm{a}$ would have improved to $1.0 \%$, while in observation $12 \mathrm{~b}$ the limit would have been $8.8 \%$.

\subsection{Verification of Procedures}

Simulated data were used to test our search codes and to verify the sensitivity of the search. As an additional check, we applied our acceleration method to SAX J1808.4-3658, the accreting millisecond pulsar (Wijnands \& van der Klis 1998; Chakrabarty \& Morgan 1998). Since our search was for a similar pulsar, with a frequency of approximately a few hundred hertz in an orbit of approximately a few hours about a low-mass companion, SAX J1808.4-3658 provides a useful test of our acceleration method.

We analyzed an $R X T E$ PCA observation (observation 30411-01-01-04). We selected energy PHA bins corresponding to $0.4-17 \mathrm{keV}$ from data of the same type we used in our search (E125). We barycenter corrected the arrival times with the position given by Roche et al. (1998) and used $2048 \mathrm{~s}$ of data beginning at Mission Elapsed Time (MET, seconds since 1994.0) 135,395,082.

The pulsations in these data are easily detected - the $\sim 401 \mathrm{~Hz}$ signal is obvious in an unaccelerated PDS, with significant power detected in each of about 30 adjacent frequency bins, using $2048 \mathrm{~s}$ of data (Fig. 1, dotted line). The highest single-bin power represents about $22 \%$ of the total signal power in the extended feature.

Using the known orbital parameters (Chakrabarty \& Morgan 1998) to remove the acceleration from the data, the

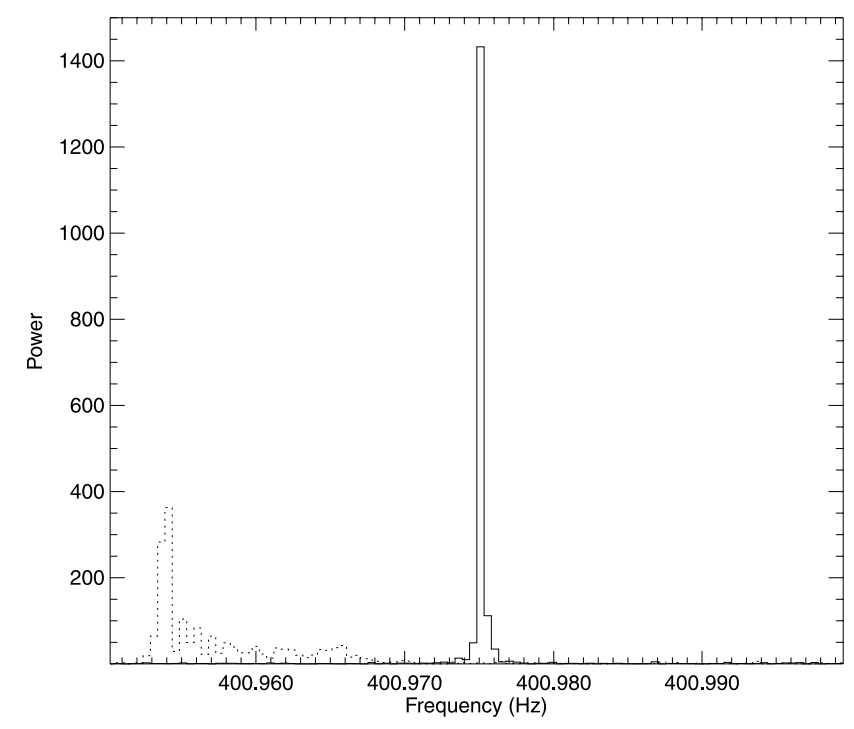

FIG. 1.-SAX J1808.4-3658. Dotted line shows a section of the unaccelerated power spectrum. Spreading of the signal power due to the orbital Doppler shift is evident. Solid line shows the power spectrum after removal of the Doppler shift using our acceleration technique. The signal power has not been confined to a single bin because the pulsar frequency lies between two discrete Fourier frequencies. During this observation, SAX J1808.4-3658 passes through longitude $=0$. Peak in uncorrected spectrum is therefore maximally offset from pulsar's rest frequency, $\Delta f_{\max }=$ $\beta f_{0}=0.022 \mathrm{~Hz}$. 
spreading of the signal power is reduced to about six independent Fourier bins around the correct rest frequency of the pulsar, $400.9752106(8) \mathrm{Hz}$, with $\sim 85 \%$ of the total power in a single spectral bin (Fig. 1, solid line). Oversampling reveals that the spreading that remains is consistent with the expected sinc-function response of the (discrete) FFT to a signal with the pulsar's rest frequency (van der Klis 1989). Thus, we confirm that our technique successfully removes the effect of the Doppler shift due to the pulsar's orbital motion.

\subsection{Search Results}

No candidate pulsations from any of the Aq1 X-1 observations exceeded our predetermined detection threshold. Using our largest detected search power, we can calculate upper limits on the strength of any pulsar signal contained in the various data sets searched (Vaughan et al. 1994), provided its orbit and spin frequency are covered by our search phase space. These upper limits are more restrictive than the a priori detection sensitivities.

The upper limit calculation is essentially the same as the detection sensitivity calculation, with the detection threshold power $P_{\text {det }}$ replaced by the maximum observed power $P_{\max }=30.96$. The results are shown in Table 3. For example, with $95 \%$ confidence, we can say that there is no sinusoidal pulsar signal in observation $10 \mathrm{a}$ with a fractional rms amplitude greater than $1.0 \%$. The limits we can place on the fractional rms become less stringent as the source flux decreases, increasing to an upper limit of $9.0 \%$ during observation 12b, when Aq1 X-1 was observed to be faintest $\left(\sim 1 \mathrm{mcrab}^{2}\right.$ 13,000 PCA counts $\left.\mathrm{s}^{-1}=1 \mathrm{crab}\right)$.

\section{DISCUSSION}

We find no evidence of pulsations from Aq1 X-1 as it fades into quiescence. The upper limits on the pulsed fraction for the eight data sets searched range from $0.52 \%$ to $9.0 \% \mathrm{rms}$. If, indeed, during the period identified as the "propeller phase" (Campana et al. 1998; Zhang et al. 1998a), the NS magnetic field significantly modifies the accretion flow geometry in the vicinity of the NS, the apparent absence of pulsations does not support the hypothesis that the quiescent emission is due to continued accretion. Our results cannot completely rule out such accretion, however, since it is possible that the geometry of the system may not lead to detectable pulsations; for example, the magnetic axis may be very nearly aligned with the rotation axis, or the rotation axis may point directly toward the Earth. The nondetection of pulsations coupled with the observation of a thermal spectrum during quiescence (Rutledge et al. 1999) favors the interpretation that the quiescent luminosity is not due to accretion but rather to a hot NS core (Brown et al. 1998).

We thank Stuart B. Anderson and Thomas A. Prince for helpful discussions. We also thank Lars Bildsten, Sergio Campana, and an anonymous referee for valuable comments on the manuscript. This work was supported by NASA grant NAG 5-3239.
Alcock, C., \& Illarionov, A. 1980, ApJ, 235, 534

Anderson, S. B., Gorham, P. W., Kulkarni, S. R., Prince, T. A., \& Wolszczan, A. 1990, Nature, 346, 42

Asai, K., Dotani, T., Kunieda, H., \& Kawai, N. 1996a, PASJ, 48, L27

Asai, K., Dotani, T., Mitsuda, K., Hoshi, R., Vaughan, B., Tanaka, Y., \& Inoue, H. 1996b, PASJ, 48, 257

Bildsten, L., Salpeter, E. E., \& Wasserman, I. 1992, ApJ, 384, 143

Brown, E. F., Bildsten, L., \& Rutledge, R. E. 1998, ApJ, 504, L95

Buccheri, R., Sacco, B., \& Ozel, M. E. 1987, A\&A, 175, 353

Campana, S., Stella, L., Mereghetti, S., Colpi, M., Tavani, M., Ricci, D., Fiume, D. D., \& Belloni, T. 1998, ApJ, 499, L65

Chakrabarty, D., et al. 1997, ApJ, 481, L101

Chakrabarty, D., \& Morgan, E. H. 1998, Nature, 394, 346

Chevalier, C., \& Ilovaisky, S. A. 1991, A\&A, 251, L11 1998, IAU Circ. 6806

Chevalier, C., Ilovaisky, S. A., Leisy, P., \& Patat, F. 1999, A\&A, 347, L51

Groth, E. J. 1975, ApJS, 29, 285

Illarionov, A. F., \& Sunyaev, R. A. 1975, A\&A, 39, 185

Leahy, D. A., Darbro, W., Elsner, R. F., Weisskopf, M. C., Kahn, S., Sutherland, P. G., \& Grindlay, J. E. 1983, ApJ, 266, 160

Markwardt, C. B., Strohmayer, T. E., \& Swank, J. H. 1999, ApJ, 512, L125

Menou, K., Esin, A. A., Narayan, R., Garcia, M. R., Lasota, J. P., \& McClintock, J. E. 1999, ApJ, 520, 276

Rajagopal, M., \& Romani, R. W. 1996, ApJ, 461, 327

Roche, P., Chakrabarty, D., Morales-Rueda, L., Hynes, R., Slivan, S. M., Simpson, C., \& Hewett, P. 1998, IAU Circ. 6885

Romani, R. W. 1987, ApJ, 313, 718

\section{REFERENCES}

Rutledge, R. E., Bildsten, L., Brown, E. F., Pavlov, G. G., \& Zavlin, V. E. 1999, ApJ, 514, 945

Shahbaz, T., Thorstensen, J. R., Charles, P. A., \& Sherman, N. D. 1998, MNRAS, 296, 1004

Smith, D. A., Morgan, E. H., \& Bradt, H. 1997, ApJ, 479, L137

Stella, L., White, N. E., \& Rosner, R. 1986, ApJ, 308, 669

Strohmayer, T. E., Jahoda, K., Giles, A. B., \& Lee, U. 1997, ApJ, 486, 355

Strohmayer, T. E., Zhang, W., Swank, J. H., Smale, A., Titarchuk, L., Day, C. \& Lee, U. 1996, ApJ, 469, L9

Swank, J. H., Jahoda, K., Zhang, W., \& Giles, A. B. 1996, in The Lives of the Neutron Stars, ed. M. A. Alpar, U. Kiziloglu, \& J. van Paradijs (NATO ASI Ser. C, 450; Boston: Kluwer), 525

van der Klis, M. 1989, in Timing Neutron Stars, ed. H. Ögelman \& E. van den Heuvel (NATO ASI Ser. C, 262; Boston: Kluwer), 27

van Paradijs, J., Verbunt, F., Shafer, R. A., \& Arnaud, K. A. 1987, A\&A, 182,47

Vaughan, B. A., et al. 1994, ApJ, 435, 362

Verbunt, F., Belloni, T., Johnston, H. M., van der Klis, M., \& Lewin, W. H. G. 1994, A\&A, 285, 903

Wijnands, R., \& van der Klis, M. 1998, Nature, 394, 344

Zavlin, V. E., Pavlov, G. G., \& Shibanov, Y. A. 1996, A\&A, 315, 141

Zavlin, V. E., Pavlov, G. G., Shibanov, Y. A., \& Ventura, J. 1995, A\&A, 297, 441

Zhang, S. N., et al. 1996, IAU Circ. 6462

Zhang, S. N., Yu, W., \& Zhang, W. 1998a, ApJ, 494, L71

Zhang, W., Jahoda, K., Kelley, R. L., Strohmayer, T. E., Swank, J. H., \& Zhang, S. N. 1998b, ApJ, 495, L9 